\title{
Does Stem Cell Regenerative Medicine Could Provide Good Clinical outcome for Deafness? -The Focus on Current Difficulties on Clinical Application
}

\author{
Subramanian Sambandam* \\ Department of Biotechnology, University of Madras, India
}

Submission: May 21, 2018; Published: July 05, 2018

*Corresponding author: Subramanian Sambandam, Department of Biotechnology, University of Madras, India,Tel: +91 63802214 99;

Email: sambandam.subramanian@gmail.com

\begin{abstract}
Genetical, ageing, excessive noise and certain antibiotics are account for majority of permanent hearing loss in humans. Hearing loss is caused by dysfunction of the sensory epithelium (the organ of Corti) within the inner ear (cochlea). It is associated with irreversible loss of sensory hair cells and spiral ganglion neurons.Inner ear hair cells are specialized mechanoreceptors converts mechanical stimuli into neural information for transmission to the brain. Several areas of research have addressed the treatment of hearing loss. There are evidences for regeneration of sensory hair cells in non-mammalian species; however, studies in mammals have failed to find evidence of cochlear hair cells regeneration.Stem cell regenerative medicine is the current focus on cure for deafness while through regeneration of specific cell types from different sources of stem cells.This review deled present stem cell regenerative therapy promising outcome on clinical application.
\end{abstract}

Keywords: Stem cell; Regenerative medicine; Deafness; Inner hair cells; Outer hair cell

Abbrevations: ICM: Inner Cell Mass; EG: Embryonic Germ; FGF: Fibroblast Growth Factor; EGF: Epidermal Growth Factor; TGF: Transforming Growth Factor; GDNF: Glial Cell-Derived Neurotrophic Factor; MSCs: Mesenchyme Stem Cells; NSCs: Neural Stem Cells; EBs: Embryoid Bodies; HES: Human Embryonic Stem Cells; SCF: Stem Cell Factor; VEGF: Vascular Endothelial Growth Factor;hBMSCs: Human Bone Marrow Stromal Cells

\section{Introduction}

Currently there is number of different approaches are underway to discover cause for sensorineural deafness. Many of the defective mechanisms that regulates the development of faulty auditory system will be discovered soon. The key importance given to mechanism that involved in specific hair cell and neural regeneration. This will provide a basic understanding of the possible ways in which auditory hair cells and nerve connection may be regenerated, thus relieving a handicap that afflicts so many people. The therapeutic management of sensorineural deafness requires maintenance, repair and regeneration of hair cells and nerve connection. The fate of any specific cell implantation requires the interaction of many different processes, including cell proliferation, migration, growth, and differentiation. Both intrinsic and extrinsic signals are required to guide cells through distinct developmental. Replacement of degenerated neurons by stem cell requires differentiation of the stem cell to the appropriate phenotype and re-establishing functional neural circuits. Neural regeneration from stem cells presents challenges in addition to the initial differentiation, such as overcoming apoptosis, preventing rejection, new neurons to grow from the cell to the target region and cochlear neurons regeneration to clinical need. Hence new methods to be identified for regeneration of hair cells and intact afferent innervations for hair cell function.

At present inner hair cell regeneration on the focus for cure for deafness. There are substantial evidences for regeneration of sensory hair cells in non-mammalian species. However, studies on mammals have failed to find evidence of cochlear hair cells regeneration. Several research strategies are currently directed towards cell transplants to restore/replace the degenerated hair cells and neural elements following hearing loss. Stem cells has the ability of prolonged selfrenewal and differentiation. This fate choice is highly regulated by intrinsic signals and the external micro-environment. Stem cells can be identified in many adult mammalian tissues, such as epithelia, blood, and germ line contribute to replenishment of cells lost through normal cellular senescence or injury. Stem 
cells present in many adult organs with more plasticity than originally thought, they typically form only a limited number of cell types. Stem cells of the early mammalian embryo, in contrast, have the potential to form any cell type. In the un-manipulated blastocyst-stage embryo, stem cells of the Inner Cell Mass (ICM) promptly differentiate in to primitive ectoderm, which ultimately differentiates during gastrulation into three Embryonic Germ (EG) layers. When removed from their normal embryonic environment and cultured under appropriate conditions, ICM cells can give rise to cells that proliferate and replace themselves indefinitely.In this present work the stem cells importance on the regeneration of hair cells and different views on the use for regeneration of inner hair cell has been discussed to understand developing new protocols for clinical application on cure for deafness.

\section{Co-Culture}

Co-culture practice gained momentum several decades in cell culture and tissue culture research techniques.The above is now widely appreciated for stem cell regenerative medicine for clinical application.The application of use of stem cells in clinical significance needs some appropriate knowledge of co-culture technique. Thus co-culture of stem cells with another cell type in order to provide cohort biochemical cues specifically required for differentiation of stem cells into a specific cell type. In vitro co-culture models provides an ideal starting point for differentiation studies in systems where little is known about the precise combination of factors that induce differentiation into a specific cell type. The interactions and signaling between developing tissues play an essential role in regulating differentiation in vivo. Co-Culture models offers methods to study differentiation under controlled conditions in in vitro with the advantage of being able to replicate some tissue derived signaling and these models has been used successfully to direct the differentiation of stem or precursor cells into neurons, hematopoietic cells, photoreceptor cells, and hepatocytes. Notably, the first published attempt to direct the differentiation of mouse embryonic stem cells toward an auditory neuron lineage used co-culture technique with early post-natal cochlear tissues to improve number of bipolar neurons.Neurotrophic factors promote the survival and differentiation of embryonic stem cells into functional neurons in in vitro. Several studies now demonstrated that, differentiation of mouse embryonic stem cells into neurons can be achieved by direct temporal exposure to growth factors and neurotrophins in a defined medium. Such studies resulted with production of neural precursors, functional post-mitotic neurons, and dopaminergic neurons following treatment with various neurotrophic agents, including basic Fibroblast Growth Factor (FGF), Epidermal Growth Factor (EGF), Transforming Growth Factor (TGF), and Glial CellDerived Neurotrophic Factor (GDNF). These studies differ in the combination and timing of growth factors treatment or application. Interestingly, embryonic stem cells can give rise to neuro-ectodermal precursor by chemically defined lowdensity culture with stromal cell-derived inducing activity.

Several stem cell types delivered into mammalian cochlea for the replacement of auditory hair cells and neurons, including bone marrow stem cells, neural stem cells and embryonic stem cells.Adult green mice bone marrow stromal cells were co-cultured with hippocampal slices differentiated to neuron-like starting at day 3 and then decreased gradually over 35 days implicate those cells ability to differentiate into neuronsand create direct contact with the host residing tissue [1]. Study investigated the functional relationship between Mesenchyme Stem Cells (MSCs) and Neural Stem Cells (NSCs) using co-culture systems demonstrated that, MSCs promoted outgrowth of NSC-derived neuritis and the majority of neuritis were found oriented parallel along the MSC axis. It is been noted that cell adhesion molecule and extracellular matrix, such as $\mathrm{N}$-cadherin, fibronectin, and laminin, contributed to this effect [2].Zhang et al.[3] examined human umbilical cord bloodderived mesenchymal stromal cells (UCB-MSCs) with Schwann cells lineage in co-culture system noted that Initially human UCB-MSCs into floating neurospheres, and then neurospheres to Schwann-like cells using glia growth factors and UCB-MSCs morphological similarities with Schwann cells. Further they noted that, differentiated UCB-MSCs could promote neurite outgrowth in coculture with dorsal root ganglia neurons. Similarly study on the interactions between Tuj-1+ bone marrow-derived MSCs and embryonic neural stem cells (NSCs) in in vitro co-culture system found MSCs with differentiating NSCs showed significant increase in Tuj-1+ neurons. Therefore, they assumed that MSCs provides instructive signals that are able to direct the differentiation of NSCs and promote axonal development in neuronal progeny [4]. Similarly highly proliferative stem cells treated with retinoic acid generated essentially pure precursors cell which identified as positive radial glaial cells and these cells generated neurons with remarkably uniform biochemical and electrophysiological characteristics [5].

Differentiation of embryonic stem cells into bipolar auditory neurons, two post-natal auditory co-culture models with and without neurotrophic support to elucidate their potential to direct the differentiation into characteristic, bipolar, auditory neurons successfully found, likely to resulted with improved clinical outcomes for cochlear implant recipients. The differentiation of stem cells into neuron-like cells was facilitated by co-culture with auditory neurons or hair cell explants isolated from post-natal day five rats noted most successful combination was the co-culture of hair cell explants with whole Embryoid Bodies (EBs), which resulted in significantly greater numbers of neurofilament-positive, neuron-like cells, [6]. It infers that coculture with partially differentiated neural cell line could be one possible clue for 
successful implantation for regeneration of hair cells in in vivo. Further, treatment of Human Embryonic Stem Cells (HES) cells during EBs development with a combination of low dose hematopoietic cytokines like Stem Cell Factor (SCF), Flt-3 ligand, Vascular Endothelial Growth Factor (VEGF) and Human Bone Marrow Stromal Cells (hBMSCs), cell clusters containing CD34-positive hematopoietic stem cells and CD45-positive mature hematopoietic cells provided the first evidence for the role of cytokine-hBMSCs combination in promoting hematopoietic differentiation [7].

In addition, the generation of viable dopamine-producing neurons from pluripotent mouse embryonic stem cells, neurotrophic factors in combination with survival-promoting factors, such as interleukin-1beta, glaial cell line-derived neurotrophic factor, neurturin, transforming growth factorbeta(3) and dibutyryl-cyclic AMP, significantly enhanced maintenance of dopaminergic neurons. Taking above experimental evidences in vitro co-cultures with controlled environment is giving satisfactory result on development of neural growth but what could be done in the case of implantation of stem cells along with appropriate supplements into the inner ear, besides, two particular technical details needs to be analyzed.

i. The embryoid bodies' development cannot be monitored in in vivo.

ii. Possibility of regeneration of neural cell types is more possible then developing into inner hair cells and find its appropriate vacant space.

Even though the neural cell type regeneration is appreciable, the appropriate connection between existing nerves is not as such easy. And another obstacle that what will happen to implanted cells is un-explainable, because, if implanted cells follow its own rule without transformation, will multiply to the extent of availability of nutrition, will occupy the tiny inner ear region and possibly will become allogeneic in nature and generate tumor. On the other side, if the implanted cells become dead and drained through lesion will also create further complication in the inner ear.Hence coculture practice in for clinical practice needs more clear and strong technological understanding on positive outcome.Even though current co-culture models provided possibility of good outcome on regeneration of inner hair cells using mitogens and successful implantation done with small rodent models proved that, implanted stem cells were able to survive only for a limited period of time.This implicate upon current co-culture methods are not well being on the clinical practice.

\section{Site of stem cell delivery}

The successful replacement of hair and supporting cells requires direct and efficient delivery of stem cells into the organ of Corti.Knowledge on the site of delivery of implantation of stem cells is the key factor for clinical application. On the above, variety of methodologies followed to deliver stem cells into the cochlea. These include:

i. Injection into semicircular canals,

ii. Adjacent to the cochlea,

iii. Direct transplantation into the cochlea

iv. Delivery into the scala media, or scala tympani, and

v. Direct injection into the auditory nerve region.

Juhn [8] noted that, transplanted cells from scala media may not able to migrate because this cavity is regulated by tight junctions that prevent implanted cell movement. Embryonic stem cells transplanted at the internal auditory meatal portion of an atrophic auditory nerve migrated extensively along it, and after four to five weeks the cells were found not only throughout the auditory nerve, but also in Rosenthal's canal and scala media, the most distal portion of the auditory nervous system where the hair cells reside. But, transplanted cell migration was more extensive in damaged auditory nerve then undamaged nerve with more signs of neuronal differentiation. This implicates the importance of tissue damage and the potential for repair [9]. In another study, bone-marrow stromal cells labeled with enhanced green fluorescent protein injected into the perilymphatic space of normal cochleae in mice. After 2 weeks it has been demonstrated that, transplanted cells settled within the cochlear tissues, especially in the spiral ligament and the spiral limbus, but most transplants were located at perilymphatic space itself [10].

In addition, the migration of stem cells into organ of Corti after delivery into the scala ty mpani demonstrated that, this has been shown to occur at low efficiency $[11,12]$, and to date there has been no evidence of these migrated cell types developing hair cell characteristics in vivo. Another major obstacle is whether transplanted cells can able to access the reticular lamina regions. The reticular lamina, which provides the tight junction barrier restricting the interchange of materials after hair cell loss due to chronic gentamycin administration demonstrated [13]. It is therefore unclear whether stem cells would be able to penetrate the reticular lamina to reach the normal location of the hair cell bodies. An important clue that stem cell migration across the reticular lamina may be possible following noise trauma caused rearrangement $[14,15]$. It is thus conceivable that during the rearrangement of these tight junctions between the formation of phalangeal scars and the restoration of the reticular lamina, transplanted stem cells would be able to cross the reticular lamina into the organ of Corti.

Furthermore, post-auricular approach to expose tympanic bulla is a well-established technique $[16,17]$. In a cochleostomy, the inner structures of the cochlea accessed by making a hole 
in the bony labyrinth [18]. The approach also has significant advantages over a lateral approach to the scala media, where it is possible to damage the stria vascularis and cochlear blood supply [19]. The direct delivery of cells into the scala media through the basilar membrane via has not been reported. It has been demonstrated that, this approach does not cause significant trauma to the cochlea. Taking above evidences, differentiation is more dependent on:

i. Area of damage

ii. Volume of damage and

iii. Damage caused by intrinsic and extrinsic factors.

The identification of the damaged area is the main object, since no appropriate protocol is identified until today.

\section{Survival and maintenance}

However, the correct target of stem cells delivery to the organ of Corti alone is unlikely to be sufficient to promote hair cell development and differentiation and also the maintenance of differentiated stem cells is another obstacle. The partially differentiated stem cells in in vitro prior to implantation may provide these cells developmental potential to form new hair cells [20]. Sakamoto et al. [20] reported the survival of stem cells predominantly in the vestibular region of the mouse inner ear and also some cells in the scala media of the cochlear duct after transplantation of four weeks. Hu et al.[21] showed that the embryonic stem cell could survive for up to nine weeks and migrated into brainstem. Neural Stem Cells (NSCs) grafted into newborn rat cochlea showed 2-4 weeks survival in the cochlear cavity and adopted the morphology and the positions of hair cells [22].

Signal transmission for the differentiation of the stem cells and its maintenance is also more important.It is established that stem cells exist in niche that provides biological signals to direct their behavior [23]. The formation of new hair cells requires pre-differentiated progenitor embryonic stem cells prior to their transplantation in in vivo, as damaged cochlear sensory epithelium may not provide all the necessary developmental signals. Though the cochlear hair cells development from neuro-ectodermal precursors is dependent on regulation of many genes and growth factors [24,25].

Some studies can be reviewed here to understand fate of implantation of stem cells with partial differentiation. Embryonic stem cells cultured in the presence of human hepato-carcinoma cell line HepG2 (MEDII medium) shown to form morphologically distinct primitive ectoderm-like cellular aggregates (EBMs) $[26,27]$. Treatment of such aggregates with Basic Fibroblast Growth Factor (bFGF) directs the cells to neuroectodermal lineage [28]. Cell types from this developmental model were selected for in vivo transplantation into the guinea pig cochlea to attempt cellular regeneration of auditory hair cells noted that, the cell types including undifferentiated embryonic stem cells and embryonic stem cells found partially differentiated in MEDII medium for three to seven days. Embryonic stem cells partially differentiated over nine days with MEDII medium and bFGF. The cells delivered into 14 guinea pigs deafened by administration of aminoglycosides prior to implantation and into one normal hearing animal demonstrated that, mouse embryonic stem cells could survive for at least nine weeks in the guinea pig cochlea and they could localize in the scala media. Study on the spiral ganglion neuron survival and dedifferentiation by application of Neurotrophic Factors (NTF), Brain Derived Neurotrophic Factor (BDNF) and Acidic Fibroblast Growth Factor (aFGF) demonstrated the significantly increased afferent peripheral process and growth of afferent nerve fiber into scala tympani and higher number of SGN densities than normal hearing animals [29]. These findings suggest embryonic stem cell types with certain degree of differentiation along with supplements may be the possible way for cell therapy approaches to regenerate cochlear hair cells. But appropriate signals are more important for differentiation into specific cell type, besides the specific cell type differentiation requires continuous seeding of signals from its environment.

More importance will be given to obstacle comes with creating artificial in vivo microenvironment. It is necessary to provide exogenous factors to induce transplanted cells to migrate, differentiate, and integrate into host cochlear tissue. Mouse embryonic stem cell types did not appear to integrate into the endogenous tissue of the guinea pig cochlea, despite the fact that, they survived within the structure for up to nine weeks because did not appear to integrate, suggests that, the remaining cells in this structure either do not elicit the appropriate biological cues to induce integration or that these signals are produced but are unable to reach the implanted embryonic stem cells due to the tight junctions that protect the cells of the organ of Corti from the contents of the perilymphatic compartments.

It is likely that, stem cell types need to be co-transplanted with appropriate factors to encourage directed differentiation and integration. It is known that neurotrophic factors are important for hair cell development [30]. Survival of Mouse Embryonic Stem Cells in the Scala Media factor (EGF) may be required to induce formation of new hair cells [31]. The formation of new hair cells also requires expression of Math1, a transcription factor downstream in the EGF pathway essential for hair cell development $[32,33]$. The regeneration of mammalian hair cells in vivo and resultant recovery of auditory function by expression of Math1 supports this combined therapeutic approach [19]. To achieve direct differentiation and integration of stem cell into new sensory hair cells necessarily requires factors like EGF and Math1. 
Even though transplantation methodology varies among publications, all authors reports the survival of exogenous stem cells in the inner ear for about 3 and 13 weeks.In addition, several of these studies report the dispersal of transplanted cells throughout the cochlea and limited detection within the target site, Rosenthal's canal. Notably, recent publication described several advances in stem cell delivery into the cochlea, including the extended survival of transplanted cells and the extensive migration along the auditory nerve. Such findings giving satisfactory results on cell transplantation therapy but many questions remain unanswered, including

i. The stage of differentiation for transplantation,

ii. The site of transplantation,

iii. Development of safe surgical techniques,

iv. Successful transplantation of exogenous cells without any rejection,

v. Existing vacant places were mostly formed scars and no reception could be expected from the scar region for new cell to bind and proceed further and

vi. Their ability to form new synapses with existing neurons. All above could be the main problems to be addressed when it comes for clinical trail but till date no particular research work carried out to resolve the above problem but perhaps many clinical practices were on board.

\section{Source of stem cell population}

Appropriate source of the stem cell applicable to the particular clinical significance is most important for achieving the desired need. On the above some information we needs to take from earlier observations. Inner ear stem cells isolation has been intensively pursued, as they seem most likely to differentiate more completely into hair cells, compared with other stem cell derivatives. Monedero et al. [34] have reported inner ear stem cell developed neuronal features and neurons differentiated from these cells grew into hair cell in in vitro. However, possibility for obtaining inner ear stem cells from human not possible or very difficult in spite of ethical reason, hence researchers can develop different stem cells derivatives from ectodermal lineage which are closer for this purpose.In birds, supporting cells within the sensory epithelia seems to be the cellular precursors for hair cell regeneration. Two precursor cell populations with a regenerative potential are currently discussed for inferior sensory epithelial damage. Cuboidal or hyaline epithelial cells appear to serve as precursors for the regeneration of both hair cells and supporting cells. For repair of superior damage, supporting cells may be the effective precursor population. With regard to fish, embryonic like neuro-epithelial cells identified as the immediate source for new hair cells and supporting cells. A large number of non- sensory supporting cells are capable of entering the cell cycle. But what stage of that cell cycle could be more appropriate for implantation is the main obstacle.Since many stem cell types are entering into cell cycle at G0/G1 phase.

Other sources like relevant neural stem cell form other vertebrates also the innovative thought. The neural stem cells are multipotential progenitor cells, characterized through the potential of self-renewal and a high plasticity to differentiate into several neuronal cell types and other germ layer tissuespecific cell lineages. Implanted neural stem cells have been shown to survive in mature cochleae of animal models and to migrate into functionally relevant regions after experimental damage to the inner ear. However, the survival of these cells in the inner ear decreases dramatically after a relatively short period. Moreover, the morphology of the implanted cells is also considered to be a critical issue. In this context, the well-established integration of transplanted neural stem cells into the organ of Corti of newborn rats, and the adoption of the morphologic phenotypes of outer or inner hair cells represent promising results, with regard to the main objective of hair cell restoration [35,36]. Furthermore, neural stem cells transplanting to the mammalian inner ear may be a source of SGN regeneration. However, neuronal differentiation is predominantly driven toward glial cell fate, rather than neurons and hair cell.As curiously noted the neural cells are non-dividing cells of the nature.

\section{Hair cell markers}

The in vitro differentiation of stem cells prior to implantation was confirmed by analysis of markers of early hair cell development and markers expressed at later stages of hair cell differentiation. The undifferentiated embryonic stem cells expressed the three hair cell markers, Myo6, a9AchR, and Myo7a, at relatively high levels when compared to the partially differentiated cell types. The embryonic stem cells were differentiated along the ectodermal lineage, the embryonic germ layer that forms the hair cells, and then to neuro-ectoderm. The specific expression pattern of the hair cell markers changed over time toward decreased expression in all three markers tested. Future studies in generating cell types with hair cell characteristics from stem cells necessarily to use alternative differentiation conditions like pathways involving Math1, shh.

\section{Immunological significance}

The generation of hair cells from stem cells is one obstacle, but immunological significance of graft is another major difficulty when it comes for implantation. Many studies have undergone to know rejection phenomenon to understand any immunological prevalence is possible for grafting.The presence of significant numbers of transplanted cells could be considered for distinct possibility for immunological response. But with animal model no significant immunological response 
was observed after implantation in guinea pig. There was no evidence of hyper acute or acute rejection in any of the immuno-suppressed guinea pigs $[37,38]$. It is also possible that the embryonic stem cells exhibit lower immunogenicity than terminally differentiated cells, although no evidence available for this. Many clinical applications for organ transplantation is done with immunosuppressive therapies but the knowledge on what extent the above therapy can be applied for stem cell transplantation for hair cell regeneration is completely not available and no particular study was devoted for the above.

\section{Conclusion}

Some animal experiments are given good understanding on using stem cells for regeneration of hair cells in vitro or in vivo. Human clinical trial using embryonic stem cells or cord blood stem cells is still on debate because of the allogenicity. In the case of bone marrow stem cells, those are adult stem cells having diversedimmunogenecity and rejection will occur, because neural cell types are mere diversed cells and are lack of many general cell markers which some time may induce autoimmunity like pattern of disease. Generally, the neural cell types are taking normal turnover very few times in its life time is another possible explanation needs to be taken in mind when those cells are going to be used for regeneration of hair cells and neural cell types.The differentiation of autologous bone marrow cells in damaged cochleae, along with their survival capacity and migrational mobility, may be exploited for the treatment of various degenerative inner ear diseases. Because most of the transplanted cells eventually evolve into non-neuronal cells. Additional studies are required to identify factors that promote the differentiation of bone marrow stem cells into distinct hair cell types and provide adequate numbers of cells that could actually enhance cochlear function. The discovery of cells displaying neuronal phenotypes in the area around the spiral ganglion is also clinically important.Mouse embryonic stem cells can differentiate into hair cells in the developing inner ear of chick embryos and into neuronal cells in the cochlea of deafened guinea pig. Adult stem cells, isolated from the mouse vestibular system, can differentiate into hair cells in the developing inner ear of chick embryos, whereas adult neural stem cells survive better and differentiate to a greater extent in deafened versus normal guinea pig cochlea. Some reports show the presence of limited numbers of nestin-positive stem cells in intact mouse organ of Corti, and dissociated neonatal rat organ of Corti, suggesting that there may be some intrinsic potential for repair. Thus, not only selection of types of stem cell is important but also what stage of the cell could be the best choice needs to be understood.

The organ of Corti poses yet another obstacle for regeneration of hair cell. If stem cells changed into outer hair cell needs to migrate outwards inner hair cells and find its correct place. The regeneration may be possible in the adult organ of Corti. The problem of hair cell regeneration is mere comparable to that of neural regeneration and even it could be considered it needs to be done in an amicable way.

\section{References}

1. Abouelfetouh A, Kondoh T, Ehara K, Kohmura E (2004) Morphological differentiation of bone marrow stromal cells into neuron-like cells after co-culture with hippocampal slice. Brain Res 1029(1): 114-119.

2. Li QM, Fu YM, Shan ZY, Shen JL, Zhang XM, et al. (2009) MSCs guide neurite directional extension and promote oligodendrogenesis in NSCs. Biochem Biophys Res Commun 384(3): 372-377.

3. Zhang HT, Cheng HY, Zhang L, Fan J, Chen YZ, et al. (2009) Umbilical cord blood cell-derived neurospheres differentiate into Schwann-like cells. Neuroreport 20(4): 354-359.

4. Croft AP, Przyborski SA (2009) Mesenchymal stem cells expressing neural antigens instruct a neurogenic cell fate on neural stem cells. Exp Neurol 216(2): 329-341.

5. Bibel M, Richter J, Schrenk K, Tucker KL, Staiger V, et al. (2004) Differentiation of mouse embryonic stem cells into a defined neuronal lineage. Nat Neurosci 7(9): 1003-1009.

6. Coleman B, Fallon JB, Pettingill LN, de Silva MG, Shepherd RK (2007) Auditory hair cell explant co-cultures promote the differentiation of stem cells into bipolar neurons. Exp Cell Res 313(2): 232-243.

7. Wang J, Zhao HP, Lin G, Xie CQ, Nie DS, et al. (2005) In vitro hematopoietic differentiation of human embryonic stem cells induced by co-culture with human bone marrow stromal cells and low dose cytokines. Cell Biol Int 29(8): 654-661.

8. Juhn SK (1998) Barrier systems in the inner ear. Acta Otolaryngol Suppl 458: 79-83.

9. Sekiya T, Kojima K, Matsumoto M, Kim TS, Tamura T, et al. (2006) Cell transplantation to the auditory nerve and cochlear duct. Exp Neurol 198(1): 12-24.

10. Sharif S, Nakagawa T, Ohno T, Matsumoto M, Kita T, et al. (2007) The potential use of bone marrow stromal cells for cochlear cell therapy. Neuroreport 18(4): 351-354.

11. Hu Z, Andang M, Ni D, Ulfendahl M (2005) Neural cograft stimulates the survival and differentiation of embryonic stem cells in the adult mammalian auditory system. Brain Res 1051(1-2): 137-144.

12. Hu Z, Wei D, Johansson CB, Holmström N, Duan M, et al. (2005) Survival and neural differentiation of adult neural stem cells transplanted into the mature inner ear. Exp Cell Res 302(1): 40-47.

13. McDowell B, Davies S, Forge A (1989) The effect of gentamicin-induced hair cell loss on the tight junctions of the reticular lamina. Hear Res 40(3): 221-232.

14. Raphael Y, Altschuler RA (1991a) Reorganization of cytoskeletal and junctional proteins during cochlear hair cell degeneration. Cell Motil Cytoskeleton 18(3): 215-227.

15. Raphael Y, Altschuler RA (1991b) Scar formation after drug-induced cochlear insult. Hear Res 51(2): 173-183.

16. Goycoolea MV, Muchow DC, Schirber CM, Goycoolea HG, Schellhas K (1990) Anatomical perspective, approach and experience with multichannel intracochlear implantation. Laryngoscope $100(2$ Pt 2 Suppl 50): 1-18.

17. Silverstein H, White DW (1990) Wide surgical exposure for singular neurectomy in the treatment of benign ositional vertigo. Laryngoscope 100(7): 701-706. 
18. Dimitrov EA, Duckert LG, Lonsbury-Martin BL, Martin GK (1988) The effect of cochleostomy on the development of endolymphatic hydrops-morphologic changes in the rabbit cochlea. Otolaryngol Head Neck Surg 98(4): 283-290.

19. Izumikawa M, Minoda R, Kawamoto K, Abrashkin KA, Swiderski DL, et al. (2005) Auditory hair cell replacement and hearing improvement by Atoh1 gene therapy in deaf mammals. Nat Med 11(3): 271-276.

20. Sakamoto T, Nakagawa T, Endo T, Kim TS, Iguchi F, et al. (2004) Fates of mouse embryonic stem cells transplanted into the inner ears of adult mice and embryonic chickens. Acta Otolaryngol Suppl 551: 48-52.

21. Hu Z, Ulfendahl M, Olivius NP (2004) Survival of neuronal tissue following xenograft implantation into the adult rat inner ear. Experimental Neurology 185(1): 7-14.

22. Ito J, Kojima K, Kawaguchi S (2001) Survival of neural stem cells in the cochlea. Acta Otolaryngol 121(2): 140-142.

23. Fuchs E, Tumbar T, Guasch G (2004) Socializing with the neighbors: stem cells and their niche. Cell 116(6): 769-778.

24. Malgrange B, Belachew S, Thiry M, Nguyen L, Rogister B, et al. (2002) Proliferative generation of mammalian auditory hair cells in culture. Mech Dev 112(1-2): 79-88.

25. Pirvola U, Ylikoski J, Trokovic R, Hebert JM, Mcconnell SK, et al. (2002) FGFR1 is required for the development of the auditory sensory epithelium. Neuron 35(4): 671-680.

26. Rathjen J, Lake JA, Bettess MD, Washington JM, Chapman G, et al. (1999) Formation of a primitive ectoderm like cell population, EPL cells, from ES cells in response to biologically derived factors. J Cell Sci 112(Pt 5): 601-612.

27. Rathjen J, Haines B, Hudson K, Nesci A, Dunn S, et al. (2002) Directed differentiation of pluripotent cells to neural lineages: homogenous formation and differentiation of a neurectoderm population. Development 129(11): 2649-2661.

28. Torres M, Girraldez F (1998) The development of the vertebrate inner ear. Mech Dev 71(1-2): 5-21.

29. Glueckert R, Bitsche M, Miller JM, Zhu Y, Prieskorn DM, et al. (2008) Deafferentation-associated changes in afferent and efferent processes in the guinea pig cochlea and afferent regeneration with chronic intrascalar brain-derived neurotrophic factor and acidic fibroblast growth factor. J Comp Neurol 507(4): 1602-1621.

30. Chabbert C, Mechaly I, Sieso V, Giraud P, Brugeaud A, et al. (2003) Voltage-gated $\mathrm{Na}+$ channel activation induces both action potentials in utricular hair cells and brain-derived neurotrophic factor release in the rat utricle during a restricted period of development. J Physiol 553(Pt 1): $113-123$

31. Doetzlhofer A, White PM, Johnson JE, Segil N, Groves AK (2004) In vitro growth and differentiation of mammalian sensory hair cell progenitors: a requirement for EGF and periotic mesenchyme. Dev Biol 272(2): 432-447.

32. Zheng JL, and Gao WQ (2000) Overexpression of Math 1 induces robust production of extra hair cells in postnatal rat inner ears. Nat Neurosci 3(6): 580-586.

33. Woods C, Montcouquiol M, Kelley MW (2004) Math1 regulates development of the sensory epithelium in the mammalian cochlea. Nat Neurosci 7(12): 1310-1318.

34. Monedero MR, Yi E, Oshima K, Glowatzki E, Edge AS (2008) Differentiation of inner ear stem cells to functional sensory neurons. Dev Neurobiol 68(5): 669-684.

35. Hughes SE, Silverman MS (1992) Explorations of otic transplantation. Exp Neurol 115(1): 37-43.

36. Kojima K, Tamura S, Nishida AT, Ito J (2004) Generation of inner ear hair cell immunophenotypes from neurospheres obtained from fetal rat central nervous system in vitro. Acta Otolaryngol Suppl (551): 2630.

37. Lin DW, Trune DR (1997) Breakdown of stria vascularis bloodlabyrinth barrier in $\mathrm{C} 3 \mathrm{H} / \mathrm{lpr}$ autoimmune disease mice. Otolaryngol Head Neck Surg 117(5): 530-534.

38. Trune DR (1997) Cochlear immunoglobulin in the C3H/lpr mouse model for autoimmune hearing loss. Otolaryngol Head Neck Surg 117(5): 504-508.

\section{Your next submission with Juniper Publishers will reach you the below assets}

- Quality Editorial service

- Swift Peer Review

- Reprints availability

- E-prints Service

- Manuscript Podcast for convenient understanding

- Global attainment for your research

- Manuscript accessibility in different formats

( Pdf, E-pub, Full Text, Audio)

- Unceasing customer service

Track the below URL for one-step submission https://juniperpublishers.com/online-submission.php 